\title{
A COMPUTATIONAL APPROACH TO PIVOT SELECTION IN THE LP RELAXATION OF SET PROBLEMS
}

\author{
F. DJANNATY AND B. ROSTAMY
}

Received 30 January 2006; Revised 17 July 2006; Accepted 10 October 2006

It has long been known to the researchers that choosing a variable having the most negative reduced cost as the entering variable is not the best choice in the simplex method as shown by Harris (1975). Thus, suitable modifications in the pivot selection criteria may enhance the algorithm. Previous efforts such as that by Dantzig and steepest-edge rules for pivot selection are based on finding a unified strategy for entering variable in all linear programming problems. In the present work, a number of strategies for pivot selection in the LP relaxation of the set problems are proposed which consider the specific knowledge of the problem. A significant reduction in the number of iterations is achieved for a set of randomly generated test problems.

Copyright (c) 2006 F. Djannaty and B. Rostamy. This is an open access article distributed under the Creative Commons Attribution License, which permits unrestricted use, distribution, and reproduction in any medium, provided the original work is properly cited.

\section{Introduction}

At the very inception of linear programming, Dantzig realized that the criterion of most negative reduced cost for selecting a new basic variable, chosen for computational ease, was not necessarily the best [5].

Many other techniques have subsequently been suggested such as "positive normalized" procedure of Dickson and Friderick [6]. Computational experiments by Wolf and Cutler [3] and kuhn and Quandt [7] showed that both the greatest change and particularly the normalized procedures were much superior to the criterion of most negative reduced cost. Since they were devised using the tableau form simplex method, they had to be discarded as impractical when the product form simplex method supersede it.

The first practical steepest-edge algorithm was developed by Harris [5] which was significantly superior to the standard simplex method. A practicable steepest-edge simplex algorithm developed by Goldfarb and Reid [7] proved to be better than reduced cost algorithm of Dantzig for updating weighted factors which are very similar to the formulas 
2 Computational approach to pivot selection

developed by kuhn and Quandt [7]. A number of other steepest-edge algorithms were presented by Forrest and Goldfarb [3] which involve both primal and dual simplexes.

\section{Set problems}

Set problems comprising set covering, set partitioning, and set packing have attracted attention for many years and have application in airline crew scheduling, bus crew scheduling, plant location, circuit switching, and information retrieval assembly line balancing [2].

Let $M=\{1,2, \ldots, m\}$ be the set of $m$ integer and let $S$ denote a set of $n$ subset of $M$. Thus

$$
\begin{gathered}
N=\{1,2, \ldots, n\}, \\
S=\left\{s_{1}, s_{2}, \ldots, s_{n}\right\} \quad \text { where } S_{j} \subseteq M, j \in N(i=1, \ldots, m, j=1, \ldots, n) .
\end{gathered}
$$

Let

$$
a_{i j}= \begin{cases}1, & i \in S_{j}, \\ 0, & i \notin S_{j} .\end{cases}
$$

The set covering problem (SCP) can be defined as follows:

$$
\begin{gathered}
\min \sum_{j=1}^{n} C_{j} x_{j}, \\
\text { s.t. } \sum a_{i j} x_{j} \geq 1 \quad(i=1, \ldots, m), \\
x_{j} \in\{0,1\} \quad(j=1, \ldots, n) .
\end{gathered}
$$

The decision variable $x_{j}$ indicates whether $s_{j}$ is selected or not and $c_{j}$ is the cost associated with selecting $s_{j}$. The problem can be interpreted as finding the minimum cost selecting of subsets of $S$.

If we replace " $\geq$ " by " $=$ " in each of the constraints of the above model, the modified problem is called the set partitioning problem (SPP). If " $\geq$ " is replaced by " $\leq$ " and the objective function is to be maximized, the resulting model is the set packing problem (SPK).

Graph theoretic relaxation of problems is an alternative way of finding quick and sharp lower bounds for set problems [2].

\section{Shortest route relaxation of the set problems}

Shortest route relaxation of the set problems as described in [1] is as follows. Each column $a_{j}$ is decomposed into $K_{j}$ arcs, where each arc corresponds to a segment of ones. If a segment of ones covers row $k$ to row $k+p$, then the associated arc runs from vertex $k$ (row index) to vertex $k+p+1$. A set of columns which constitute the shortest route from vertex 1 to vertex $m+1$ defines a feasible solution to the SCP(SPP). Row $m+1$ which 
corresponds to vertex $m+1$ is identical to row $m$. The SCP can be written as

$$
\begin{gathered}
\min \sum_{j=1}^{n} C_{j} X_{j}, \\
\text { s.t. } \sum_{j=1}^{n} a_{i j} X_{j} \geq 1 \quad(i=1, \ldots, m), \\
x_{j} \in\{0,1\} \quad(j=1, \ldots, n) .
\end{gathered}
$$

Let $H_{j}^{\prime}=\left\{i \mid a_{i j}=1, a_{i-1, j}=0, i \in\{1,2, \ldots, m\}\right\}$ where $a_{0 j}=0$. Let $K_{j}=\left|h_{j}^{\prime}\right|$ denote the number of segments of arcs in column $a_{j}$. Let SP define the cardinality of segment $P$ in column $a_{j}$ where $P=\{1, \ldots, K\}$. Let $S_{j}$ denote the index set of the segment cardinality for the column $a_{j}$, such that

$$
S_{j}=\left\{S_{1}, \ldots, S_{k_{j}}\right\}
$$

Let $H_{j}^{\prime}$ be reexpressed as $H_{j}^{\prime}=\left\{i_{1}, i_{2}, \ldots, i_{k_{j}}\right\}$. Introduce the vertex set $V$ corresponding to the rows $i=1, \ldots, m, m+1$ such that

$$
A_{j}=\left\{\left(v_{i_{1}}, v_{i_{1}+s_{1}}\right), \ldots,\left(v_{i_{k}}, v_{i_{k_{j}}+s_{k_{j}}}\right)\right\}, \quad j=1, \ldots, n .
$$

Let the associated cost for each arc in the arc set $A_{j}$ be defined as

$$
d_{p q}^{j}=\frac{(q-p) c_{j}}{\left|H_{j}\right|} \quad \text { such that } \sum_{\left(v_{p}, v_{q}\right) \in A_{j}} d_{p q}^{j}=c_{j} .
$$

Note that this is only one cost allocation strategy. A number of strategies for the shortest route relaxation of the set covering problem are proposed in [2]. The role of the row counts and column counts in upgrading the cost allocation strategy for set problems is emphasized.

Based on this work, allocating small cost to rows having small row counts in the cost allocation strategy enhances the shortest route relaxation of the set problems. We were motivated to apply a similar strategies involving row counts and column counts to the LP relaxation of the set problems. It turned out that some of these strategies can be applied to the LP relaxation of these problems after some modifications. In order to evaluate the proposed strategies a number of test problems were generated randomly whose details are discussed in Section 4.

\section{Problem-specific knowledge}

Consider the linear programming problem

$$
\begin{gathered}
\max Z=C X, \\
\text { s.t. } A X=b, \\
X \geq 0,
\end{gathered}
$$


where $A$ is a matrix of order $m \times n, b$ is a column vector $m \times 1$, and $C$ is a row vector $1 \times n$. George B. Dantzig developed simplex method which deals with the linear programming problems. Simplex method is an iterative process where in each iteration the algorithm moves from one extreme point to an adjacent extreme point with a better objective function. This move involves selecting a nonbasic variable as entering variable, selecting a basic variable as leaving variable, and replacing it by the entering variable. Dantzig rule of pivot selection involves choosing the column of the most negative reduced cost. This criterion uses information obtained from the cost row $(C)$, right-hand sides, and the pivot column. As only a small part of the problem information is utilized and problem specific knowledge is not considered, this criterion is not the best strategy for the pivot selection. Harris [5] has made a significant effort to use some information about technological coefficients of the problem and reported promising results. He proposes a general strategy for pivot selection; however, the problem specific knowledge is not fully utilized.

We will show that a pivot selection strategy may work well for a particular class of problems while it may not be suitable for another problem instance.

Linear programming is extensively used in solving integer programming problem and most successful approaches to IP problem are usually based on linear programming. In this paper, pivot selection in an important class of problems, namely, set problem is investigated.

\section{Pivot selection strategies}

Let $\mathbf{R}$ be the set of indices of the nonbasic variables in the linear programming problem, $c_{j}$ the coefficient of the original variable $x_{j}$, in the initial tableau, $\bar{c}_{j}$ is the coefficient of $x_{j}$ in row zero of other iterations (i.e., $\bar{c}_{j}=z_{j}-c_{j}$ ), and $R_{r}=\left\{j \in R \mid \bar{C}_{j}<0\right\}$.

Strategy 1 . The relation $Z=C_{B} B^{-1} b$, where $B$ is the current basis, can justify the proportionality of the norm with $\bar{C}_{j}$ and $b_{i}$ in the numerator. As the number of nonzeros in a column is restrictive and imposes some restriction on each equation where it has nonzero coefficient then there should be some inverse proportionality to $h_{j}$.

Let $j \in R_{r}$, then we define

$$
\operatorname{norm}(j)=\frac{\left(\bar{C}_{j}\right)^{2} \times \sqrt{\sum_{i=1}^{m} a_{i j} b_{i}^{2}}}{\sqrt{1+h_{j}^{2}}}
$$

where $h_{j}$ is the number of nonzero entries in column $j$. A column having the largest norm is selected as the pivot column.

Strategy 2. The importance of row counts in enhancing the shortest route relaxation of the set covering problem is investigated in [1]. We were motivated to apply the same approach to the LP relaxation of set problems. One way of showing the restrictive effect of selecting pivot column on the linear system of equations $A X=b$ is by involving row count in the pivot selection. Row count of each row reflects the number of variables that are affected by choosing one of the nonbasic variables appearing in that row with a positive coefficient to enter the basis. 
Let $j \in R_{r}$ and let $f_{i}$ be the number of nonzero entries in row $i$, then for $(i=1, \ldots, m)$ we define

$$
\operatorname{norm}(j)=\frac{\left(\bar{C}_{j}\right)^{2} \times \sqrt{\sum_{i=1}^{m} a_{i j} b_{i}^{2}}}{\sqrt{1+\sum_{i=1}^{m} a_{i j}(\operatorname{rowcount}(i))^{2}}} .
$$

A column having the largest norm is selected as the pivot column.

Strategy 3. Let $j \in R_{r}$, then we define

$$
\operatorname{norm}(j)=\frac{\bar{C}_{j}}{\sqrt{1+\sum_{i=1}^{m} a_{i j}(\operatorname{rowcount}(i))^{2}}} .
$$

A column having the largest norm is selected as the pivot column.

Strategy 4. Let $j \in R_{r}$, then we define

$$
\operatorname{norm}(j)=\frac{\bar{C}_{j}}{1+h_{j}^{2}}
$$

A column having the largest norm is selected as the pivot column.

Strategy 5. Let $j \in R_{r}$, then we define

$$
\operatorname{norm}(j)=\frac{\left(\bar{C}_{j}\right)^{2}}{\left(1+h_{j}^{2}\right) \times\left(\sqrt{1+\sum_{i=1}^{m} a_{i j}(\operatorname{rowcount}(i))^{2}}\right)} .
$$

A column having the largest norm is selected as the pivot column.

Strategy 6. Let $j \in R_{r}$, then we define

$$
\operatorname{norm}(j)=\frac{\bar{C}_{j}}{\sqrt{1+\sum_{i=1}^{m} a_{i j} e^{\text {rowcount }(i)}}}
$$

A column having the largest norm is selected as the pivot column.

Strategy 7. Let $j \in R_{r}$, then we define

$$
\operatorname{norm}(j)=\operatorname{random}\left(\bar{C}_{j}\right)
$$

As noted in [8] the effect of parameters $C, B$, and $A$ is investigated. 
Table 6.1. SCP problem.

\begin{tabular}{lcc}
\hline & SCP1, $200 \times 200$ & \\
\hline Run time & No. of iterations & Strategy \\
\hline 3.15 & 5195 & Dantzig (P) \\
1.20 & 2399 & Strategy 1 \\
3.45 & 465 & Strategy 3 \\
0.24 & 450 & Strategy 4 \\
1.50 & 354 & Strategy 5 \\
3.37 & 450 & Strategy 6 \\
3.31 & 5358 & Strategy 7 \\
\hline
\end{tabular}

\section{Computational results}

6.1. Test problems. Avis and Chavtal [6] have generated a class of LP problems which are used as test problems. The general form of these problems is as follows:

$$
\begin{gathered}
\max \sum_{j=1}^{n} X_{j}, \\
\text { s.t. } \sum_{j=1}^{n} a_{i j} X_{j} \leq 10^{4} \quad(i=1, \ldots, m), \\
X_{j} \geq 0 \quad(j=1, \ldots, n) .
\end{gathered}
$$

The only parameters which have to be specified are $a_{i j}$ 's which are taken as random integers in the interval $[1,1000]$.

Inspired by the random model proposed by Avis and Chavtal, we used a similar model to create our test problems which can be described as follows:

$$
\begin{gathered}
\min \sum_{j=1}^{n} C_{j} X_{j}, \\
\text { s.t. } \sum_{j=1}^{n} a_{i j} X_{j} \geq 1 \quad(i=1, \ldots, m), \\
0 \leq X_{j} \leq 1 \quad(j=1, \ldots, n),
\end{gathered}
$$

where $a_{i j}$ 's are binary random integers, and $C_{j}$ 's are random integers, taken in the interval $[1,100]$. The smallest and largest problems considered are $10 \times 10$ and $500 \times 700$, respectively. The reason that $a_{i j}$ 's are binary is that we are dealing with set problems.

As can be seen from Tables 6.1, 6.2, 6.3, and 6.4, all strategies except for Strategy 2 are better than Dantzig rule both in the sense of the number of iterations and in the sense of execution times. Strategy 5 has reduced the number of iterations by 40 times which causes a significant reduction in round-off errors. As it is expected, random strategy is the worst in the sense of the number of iterations.

Computational result for SPP problems. As can be seen in Tables 6.5, 6.6, 6.7, and 6.8 Strategy 1 is better than other strategies, both in the number of iterations and in the sense 
Table 6.2. SCP problem.

\begin{tabular}{lcc}
\hline & SCP2 $250 \times 300$ & \\
\hline Run time & No. of iterations & Strategy \\
\hline 11.40 & 10196 & Dantzig (P) \\
2.15 & 1995 & Strategy 1 \\
7.45 & 564 & Strategy 3 \\
0.49 & 524 & Strategy 4 \\
4.50 & 324 & Strategy 5 \\
8.12 & 524 & Strategy 6 \\
11.23 & 9976 & Strategy 7 \\
\hline
\end{tabular}

Table 6.3. SCP problem.

\begin{tabular}{lcc}
\hline & CP3 $350 \times 500$ & \\
\hline Run time & No. of iterations & Strategy \\
\hline 45.20 & 16124 & Dantzig (P) \\
11.20 & 3947 & Strategy 1 \\
20.35 & 743 & Strategy 3 \\
2.20 & 701 & Strategy 4 \\
21.00 & 452 & Strategy 5 \\
24.30 & 701 & Strategy 6 \\
46.50 & 17112 & Strategy 7 \\
\hline
\end{tabular}

Table 6.4. SCP problem.

\begin{tabular}{lcc}
\hline & SCP4, $500 \times 700$ & \\
\hline Run time & No. of iterations & Strategy \\
\hline 66.15 & 23839 & Dantzig (P) \\
34.30 & 7411 & Strategy 1 \\
41.23 & 1416 & Strategy 3 \\
8.27 & 1381 & Strategy 4 \\
39.44 & 981 & Strategy 5 \\
45.56 & 1381 & Strategy 6 \\
69.58 & 25912 & Strategy 7 \\
\hline
\end{tabular}

Table 6.5. SPP problem.

\begin{tabular}{lcc}
\hline & SPP1, $200 \times 200$ & \\
\hline Run time & No. of iterations & Strategy \\
\hline 0.30 & 1359 & Dantzig (P) \\
0.15 & 372 & Strategy 1 \\
0.50 & 317 & Strategy 2 \\
0.52 & 352 & Strategy 5 \\
0.41 & 1590 & Strategy 7 \\
\hline
\end{tabular}


8 Computational approach to pivot selection

Table 6.6. SPP problem.

\begin{tabular}{lcc}
\hline & SPP2, $250 \times 300$ & \\
\hline Run time & No. of iterations & Strategy \\
\hline 1.14 & 1712 & Dantzig (P) \\
0.37 & 637 & Strategy 1 \\
2.40 & 555 & Strategy 2 \\
4.48 & 1150 & Strategy 5 \\
1.27 & 1910 & Strategy 7 \\
\hline
\end{tabular}

Table 6.7. SPP problem.

\begin{tabular}{ccc}
\hline & SPP3, 350 $\times 500$ & \\
\hline Run time & No. of iterations & Strategy \\
\hline 7.02 & 4187 & Dantzig (P) \\
3.01 & 1605 & Strategy 1 \\
22.37 & 1594 & Strategy 2 \\
27.39 & 2011 & Strategy 5 \\
8.11 & 4980 & Strategy 7 \\
\hline
\end{tabular}

Table 6.8. SPP problem.

\begin{tabular}{ccc}
\hline & SPP4 $500 \times 700$ & \\
\hline Run time & No. of iterations & Strategy \\
\hline 42.15 & 24971 & Dantzig (P) \\
16.47 & 7983 & Strategy 1 \\
89.14 & 7251 & Strategy 2 \\
102.44 & 11298 & Strategy 5 \\
53.13 & 27356 & Strategy 7 \\
\hline
\end{tabular}

Table 6.9. SPK problem.

\begin{tabular}{lcc}
\hline & SPK1, $200 \times 200$ & \\
\hline Run time & No. of iterations & Strategy \\
\hline 0.34 & 1558 & Dantzig (P) \\
0.27 & 516 & Strategy 1 \\
1.30 & 560 & Strategy 2 \\
2.12 & 610 & Strategy 5 \\
0.42 & 1743 & Strategy 7 \\
\hline
\end{tabular}

of execution time. Strategy 7 is the worst because it is based on the random selection of the pivot columns.

Computational result for SPK problems. As can be seen in Tables 6.9, 6.10, 6.11, 6.12 Strategy 1 is better than all other strategies including Dantzig rule in the sense of iteration number and execution times. 
Table 6.10. SPK problem.

\begin{tabular}{lcc}
\hline & SPK2, $250 \times 300$ & \\
\hline Run time & No. of iterations & Strategy \\
\hline 1.33 & 2374 & Dantzig (P) \\
0.54 & 872 & Strategy 1 \\
2.51 & 911 & Strategy 2 \\
3.23 & 873 & Strategy 5 \\
1.58 & 2691 & Strategy 7 \\
\hline
\end{tabular}

Table 6.11. SPK problem.

\begin{tabular}{lcc}
\hline & SPK3, $350 \times 500$ & \\
\hline Run time & No. of iterations & Strategy \\
\hline 9.33 & 5077 & Dantzig (P) \\
5.2 & 2808 & Strategy 1 \\
11.4 & 2914 & Strategy 2 \\
12.43 & 2863 & Strategy 5 \\
10.29 & 5492 & Strategy 7 \\
\hline
\end{tabular}

Table 6.12. SPK problem.

\begin{tabular}{lcc}
\hline \multicolumn{3}{c}{ SPK4, $500 \times 700$} \\
\hline Run time & No. of iterations & Strategy \\
\hline 47.11 & 21349 & Dantzig (P) \\
21.34 & 5013 & Strategy 1 \\
44.28 & 6143 & Strategy 2 \\
50.35 & 5493 & Strategy 5 \\
52.22 & 23982 & Strategy 7 \\
\hline
\end{tabular}

\section{Comparison with a variant of steepest edge}

In this section we review the primal steepest-edge algorithm proposed in [4] by Goldfarb and Reid for solving the standard form linear programming problem:

$$
\begin{aligned}
& \operatorname{minimize} c^{T} x, \\
& \text { subject to } A x=b, \\
& \qquad x \geq 0,
\end{aligned}
$$

where $A$ is an $m \times n$ matrix of rank $m$ and $m<n$.

Consider a single step of the simplex method applied to $(*)$ and let $B$ and $N$ denote the submatrices of $A$ corresponding to basic and nonbasic columns, respectively. To simplify 
Table 7.1

\begin{tabular}{lccccccccc}
\hline SCP & Dantzig & St1 & St2 & St3 & St4 & St5 & St6 & St7 & Ried \\
\hline $30 \times 30$ & 97 & 46 & 60 & 51 & 49 & 50 & 50 & 91 & 84 \\
$200 \times 200$ & 4601 & 1512 & 2911 & 394 & 259 & 611 & 324 & 3491 & 779 \\
$250 \times 300$ & 4102 & 1881 & 2974 & 566 & 519 & 413 & 521 & 7196 & 783 \\
$350 \times 500$ & 15914 & 4721 & 7119 & 814 & 769 & 601 & 764 & 15113 & 1162 \\
$500 \times 700$ & 21409 & 6013 & 11146 & 1410 & 1416 & 1201 & 1422 & 24917 & 1587 \\
\hline
\end{tabular}

Table 7.2

\begin{tabular}{lccccccccc}
\hline SPP & Dantzig & St1 & St2 & St3 & St4 & St5 & St6 & St7 & Ried \\
\hline $30 \times 30$ & 32 & 27 & 26 & 57 & 49 & 23 & 66 & 40 & 30 \\
$200 \times 200$ & 792 & 371 & 311 & - & - & 357 & - & 894 & 686 \\
$250 \times 300$ & 1712 & 637 & 555 & - & - & 1150 & - & 1816 & 1612 \\
$350 \times 500$ & 4187 & 1605 & 1594 & - & - & 2011 & - & 5041 & 7870 \\
$500 \times 700$ & 24971 & 7983 & 7251 & - & - & 11298 & - & 28432 & 10327 \\
\hline
\end{tabular}

our exposition, we will henceforth assume that the first $m$ columns of $A$ and components of $x$ are basic at the start of a step. This step is of the form

$$
\bar{x}=x+\theta \eta_{q}, \quad \text { where } x=\left[\begin{array}{c}
B^{-1} b \\
0
\end{array}\right] .
$$

The basic feasible solution at the start of the step, $\eta_{q}$, is one of the set of edge directions

$$
\eta_{j}=\left[\begin{array}{c}
-B^{-1} N \\
I
\end{array}\right] e_{j-m}, \quad j=m+1, \ldots, n
$$

Emanating from the vertex $x$, and $\theta$ is the length of the step, $e_{i}$ denotes the $i$ th column of the identity matrix $I$. The edge direction $\eta_{q}$ must be "downhill," that is, $\eta_{q}$ must make an obtuse angle with the gradient $c$ of objective function, or equivalently, the reduced cost $\bar{c}_{q}=c^{T} \eta_{q}$ must be negative. In the steepest-edge simplex algorithm, the edge $\eta_{q}$ is chosen, such that

$$
\frac{c^{T} \eta_{q}}{\left\|\eta_{q}\right\|}=\min _{j>m}\left\{\frac{c^{T} \eta_{j}}{\left\|\eta_{j}\right\|}\right\} .
$$

Tables 7.1, 7.2, and 7.3 present a comparison of our strategies with the steepest edge of Goldfarb and Reid.

As previously mentioned Strategies 3 and 4 are not strong enough to be included in the above-mentioned tables. It can be concluded that the strategies mentioned in the paper are superior to this variant of the steepest edge. 
Table 7.3

\begin{tabular}{lccccccccc}
\hline SPK & Dantzig & St1 & St2 & St3 & St4 & St5 & St6 & St7 & Ried \\
\hline $30 \times 30$ & 21 & 19 & 26 & 77 & 60 & 25 & 74 & 28 & 28 \\
$200 \times 200$ & 1589 & 874 & 1167 & - & - & 703 & - & 1873 & 735 \\
$250 \times 300$ & 2374 & 827 & 911 & - & - & 873 & - & 2691 & 1074 \\
$350 \times 500$ & 5077 & 2808 & 2914 & - & - & 2863 & - & 5492 & 3548 \\
$500 \times 700$ & 21349 & 5013 & 6143 & - & - & 5493 & - & 23982 & 8119 \\
\hline
\end{tabular}

\section{Conclusion}

A number of strategies were proposed for the pivot selection in the LP relaxation of the set problems. It is demonstrated that considering problem specific knowledge in pivoting in the LP relaxation of the set problems can enhance pivot selection in the simplex method for each instance of the set problems. one of the strategies works better than the others. Researchers in the future may come up with new strategies for particular instances of LP problems which considerably enhance pivot selection in the simplex method.

\section{References}

[1] F. Djannaty, Network based heuristics for the set covering problem, Ph.D. thesis, Department of Mathematics and Statistic, Brunel University, Brunel, 1997.

[2] - Some useful cost allocation strategies for the shortest route relaxation of the set covering problem, Electronic Journal of SADIO 22 (2002), no. 5, 12-23.

[3] J. J. Forrest and D. Goldfarb, Steepest-edge simplex algorithms for linear programming, Mathematical Programming 57 (1992), no. 3, 341-374.

[4] D. Goldfarb and J. K. Reid, A practicable steepest-edge simplex algorithm, Mathematical Programming 12 (1977), no. 3, 361-371.

[5] P. M. J. Harris, Pivot selection methods of the Devex Lp code, Computational Practice in Mathematical Programming, Mathematical Programming Studies, vol. 4, North-Holland, Amsterdam, 1975, pp. 30-57.

[6] B. L. Kaluzny, Finite Pivot algorithms and feasibility, Msc thesis, School of Computer Science, McGill University, Quebec, 2001.

[7] H. W. Kuhn and R. E. Quandt, On upper bound for the number of iterations in solving linear Programs, Operations Research 12 (1964), no. 1, 161-165.

[8] B. Rostamy, Pivot selection strategies in simplex method, Msc thesis, University of Kurdistan, Kurdistan, 2005.

F. Djannaty: Department of Mathematics, Faculty of Science, Kurdistan University,

P.O. Box 416, Sanandaj 66177, Iran

E-mail address: fdjanaty@uok.ac.ir

B. Rostamy: Department of Mathematics, Faculty of Science, Kurdistan University,

P.O. Box 416, Sanandaj 66177, Iran

E-mail address: brorzou_rostami@yahoo.com 


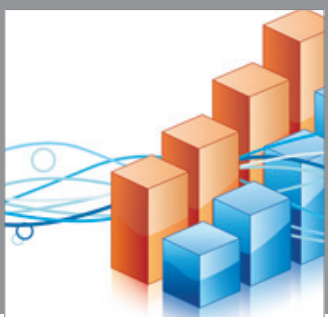

Advances in

Operations Research

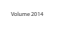

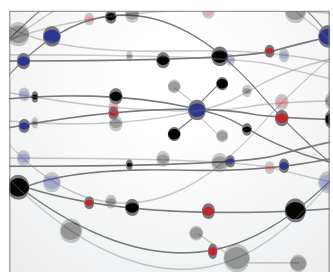

\section{The Scientific} World Journal
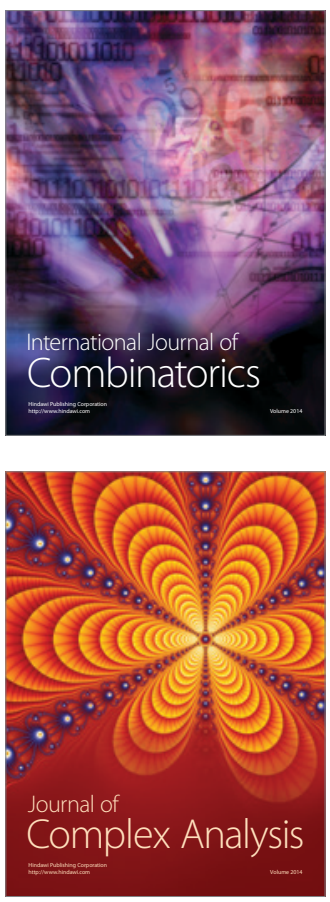

International Journal of

Mathematics and

Mathematical

Sciences
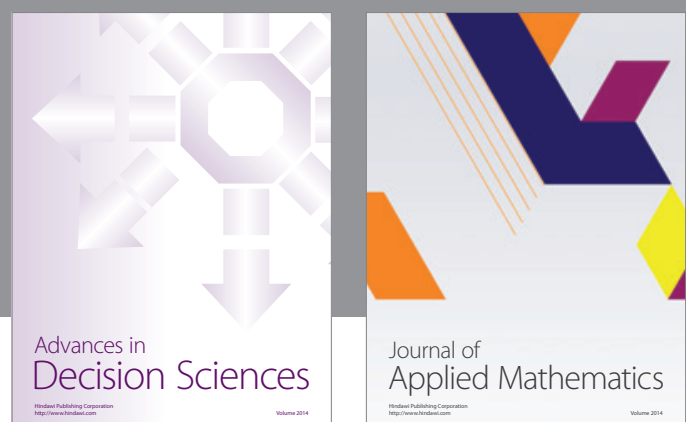

Journal of

Applied Mathematics
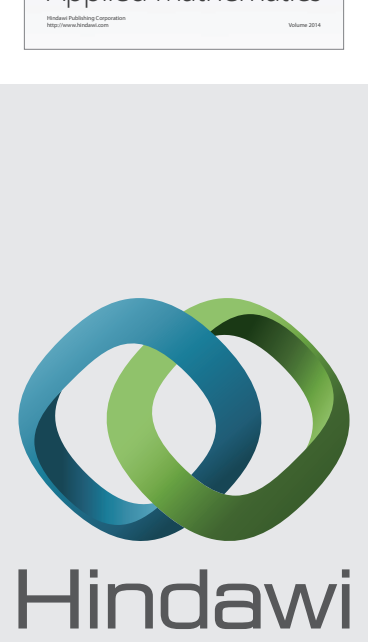

Submit your manuscripts at http://www.hindawi.com
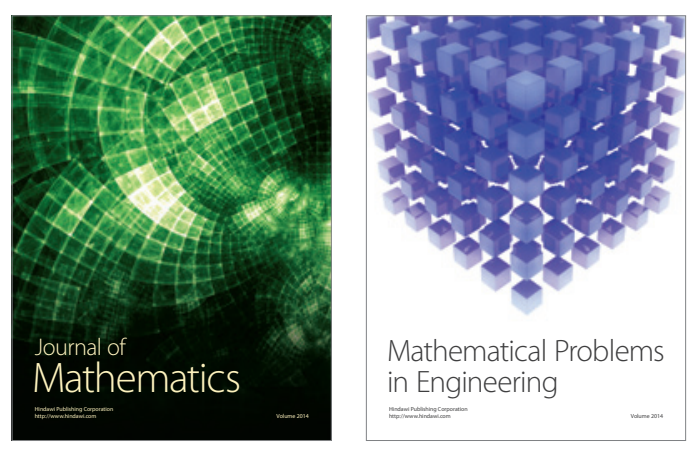

Mathematical Problems in Engineering
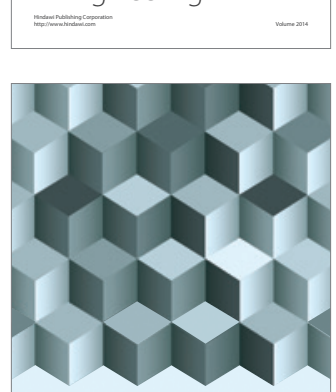

Journal of

Function Spaces
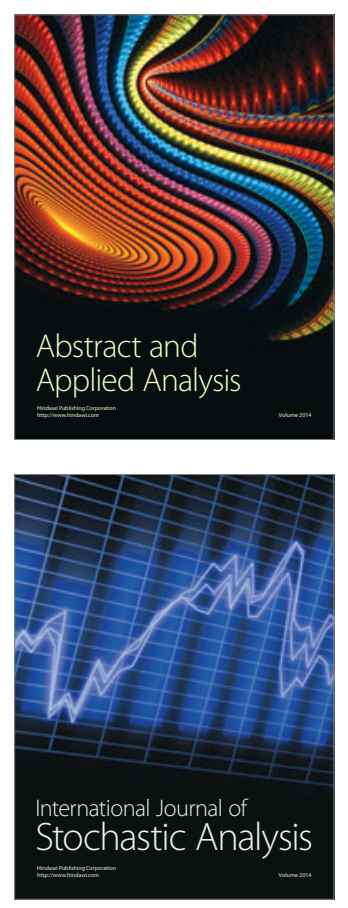

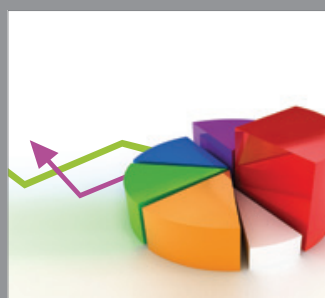

ournal of

Probability and Statistics

Promensencen
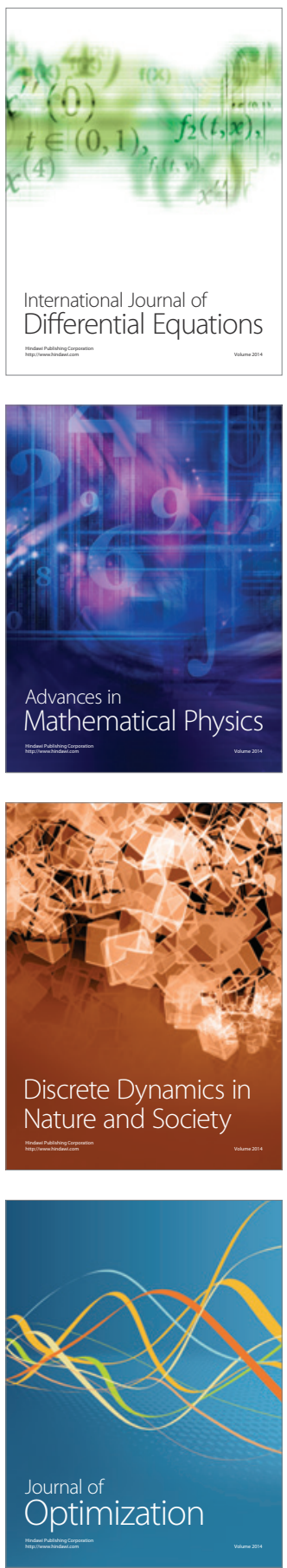